\title{
A Feature-Extraction-Based Lightweight Convolutional and Recurrent Neural Networks Adaptive Computing Model for Container Terminal Liner Handling Volume Forecasting
}

\author{
Bin Li $\mathbb{D}^{1}$ and Yuqing $\mathrm{He} \mathbb{B}^{2}$ \\ ${ }^{1}$ School of Mechanical \& Automotive Engineering, Fujian University of Technology, Fuzhou 350118, China \\ ${ }^{2}$ School of Transportation, Fujian University of Technology, Fuzhou 350118, China \\ Correspondence should be addressed to Bin Li; mse2007_lb@whut.edu.cn
}

Received 12 July 2021; Accepted 17 August 2021; Published 27 August 2021

Academic Editor: Jianfeng Zheng

Copyright (c) $2021 \mathrm{Bin} \mathrm{Li}$ and Yuqing He. This is an open access article distributed under the Creative Commons Attribution License, which permits unrestricted use, distribution, and reproduction in any medium, provided the original work is properly cited.

\begin{abstract}
The synergy of computational logistics and deep learning provides a new methodology and solution to the operational decisions of container terminal handling systems (CTHS) at the strategic, tactical, and executive levels. Above all, the container terminal logistics tactical operational complexity is discussed by computational logistics, and the liner handling volume (LHV) has important influences on a series of terminal scheduling decision problems. Subsequently, a feature-extraction-based lightweight convolutional and recurrent neural network adaptive computing model (FEB-LCR-ACM) is presented initially to predict the LHV by the fusion of multiple deep learning algorithms and mechanisms, especially for the specific feature extraction package of tsfresh. Consequently, the container-terminal-oriented logistics service scheduling decision support design paradigm is put forward tentatively by FEB-LCR-ACM. Finally, a typical large-scale container terminal of China is chosen to implement, execute, and evaluate the FEB-LCR-ACM based on the terminal running log around the indicator of LHV. In the case of severe vibration of LHV between 2 twenty-foot equivalent units (TEUs) and 4215 TEUs, while forecasting the LHV of 300 liners by the log of five years, the forecasting error within 100 TEUs almost accounts for $80 \%$. When predicting the operation of 350 ships by the log of six years, the forecasting deviation within 100 TEUs reaches up to nearly 90\%. The abovementioned two deep learning experimental performances with FEB-LCR-ACM are so far ahead of the forecasting results by the classical machine learning algorithm that is similar to Gaussian support vector machine. Consequently, the FEB-LCR-ACM achieves sufficiently good performance for the LHV prediction with a lightweight deep learning architecture based on the typical small datasets, and then it is supposed to overcome the operational nonlinearity, dynamics, coupling, and complexity of CTHS partially.
\end{abstract}

\section{Introduction}

As the traditional, large-scale logistics warehousing hub, the production plan, task scheduling, resource allocation, and execution control of container terminal handling systems (CTHS), which together are referred to as PSAC, have been the classical nondeterministic polynomial complete (NPC) problems even for the single job stage at container terminals. Those have been the hotspots and difficulties whether in the operations research theory or in the logistics industry production practice [1-11]. The classical methods, which cover operational programming [12], optimization algorithm [13], system simulation $[14,15]$, and simulation-based optimization [16], have not made a fundamental breakthrough in solving the above problems; moreover, they are increasingly struggling to cope with the myriad new problems that are constantly emerging in the logistics industry.

Fortunately, the emergence, development, and application of computational thinking [17-20] and deep learning [21-24] provide the new theory, approach, and solution to deal with the operational scheduling and decision support of CTHS at all strategic, tactical, and executive levels. In particular, the recurrent neural network (RNN) and convolutional neural network (CNN) have been widely used in various scenarios of deep learning, such as image 
recognition [25], natural language processing [26], biomedical processing [27], stock market [28], machine health monitoring [29], intersectional traffic [30], and so on. However, both are seldom applied in the production and service operations. A feature-extraction-based lightweight convolutional and recurrent neural networks adaptive computing model (FEB-LCR-ACM) is proposed for the prediction of liner handling volume (LHV) at container terminals, which is a central running index of CTHS for both carriers and terminals. It is supposed to elaborate and demonstrate the solution to overcome the parallelism, dynamics, nonlinearity, coupling, context-sensitivity, and complexity (PDN-CCC) of CTHS, which is at least partly valid and available, by the combination and integration of computational thinking and deep learning.

The rest of the paper is organized as follows: In Section 2, the applications of machine learning and deep learning in logistics production and service field are systematically reviewed, and the research trend of deep learning is prospected in the logistics industry, especially for CTHS. Section 3 sets forth the container terminal logistics tactical operational complexity, and draws the outline of the solution by the fusion of computational logistics and deep learning. Section 4 proposes FEB-LCR-ACM in particular to support the planning, scheduling, control, and execution of container-terminal-oriented logistics generalized computation abstraction, automation, and intelligence. A real case study of CTHS is intended to implement the index forecasting and performance evaluation of LHV by the classical machinelearning algorithm and FEB-LCR-ACM in Section 5. Finally, conclusions and suggestions for future research are given in Section 6.

\section{Literature Review and Related Works}

With the rapid development of the logistics industry and the leaps and bounds of machine learning, the combination between the two are increasingly close, especially for the deep learning for complex logistics systems (CLS). The literature review is divided into two main parts. The first part refers to literature review that the machine learning and deep learning are applied in the logistics and supply chain key service indicators forecasting. The second part refers to literature review that the machine learning and deep learning are put into use in the operating decision support of CLS, especially for CTHS.

In the past five years, artificial intelligence and big data have been gradually applied to supply chain management and logistics operational decision; furthermore, this trend is more and more obvious in applied theoretical research or industrial engineering practice. Monostori et al. pointed out the deficiencies and limitations of the classical applications of control engineering and information technology in production and logistics [31]. Thereupon, for one thing, the machine learning and deep learning are introduced to forecast the logistics service key indicators at the tactical and executive level, which aim to carry out good operational planning. Chung et al. concisely examined data science and analytics in aviation studies in several critical areas, namely, big data analysis, air transport network management, forecasting, and machine learning [32]. A novel, deep belief network method was employed to mine the inner patterns of flight delays, and supervised fine-tuning was performed together with support vector regression by $\mathrm{Yu}$ et al. [33]. A deep learning with one-step integration optimal decisionmaking approach based CNN and long short-term memory (LSTM) was proposed to intelligently integrate inventory optimization and demand-forecasting process during crossborder e-commerce by Ren et al. [34]. Punia et al. proposed a novel forecasting method that combines the deep learning method-LSTM networks and random forest to model complex relationships of both temporal and regression type, which were evaluated on a real-world multivariate dataset from a multi-channel retailer [35]. Shen and Lin compared two classes of the state-of-the-art deep learning methods in their ability to predict short-term crowd-shipping delivery trip production. One captured only the temporal features, and the other considered both spatial and temporal features [36]. Chen et al. raised the gradient boosting partitioned regression tree model to forecast freight travel time based on big data collected from the traffic Internet of Things infrastructure [37]. Guo et al. [38] and Huang et al. [39] designed and implemented regional logistics demand forecasting by multilayer perceptron and back propagation neural network, respectively. Petros and Ken came up with three methods of developing the Bayesian networks employed for spare parts demand forecasting [40]. Thorben and Till investigated how a network model with a stochastic block of interconnections was applied to model and predict material flows in manufacturing systems [41]. In particular, Pani et al. presented some preliminary results obtained using data mining and proposed a classification and regression trees model to reduce the range of uncertainty of ship arrivals in port in the past [42]. Recently, Gao et al. trained the LSTM RNN to predict daily volumes of containers, which entered the storage yard, by deep learning the historical dataset [43]. The LSTM networks were also implemented to forecast container throughput by Shankar et al.; moreover, the container throughput data of the Port of Singapore were used for empirical analysis [44].

Machine learning and deep learning are gradually applied into the production scheduling and executive decisions support except for forecasting key service indicators. Liu stated explicitly that the high-quality development of the logistics industry needed to realize machine learning and embedded systems [45]. In consequence, Liu et al. put forward a framework to estimate large-scale logistics network parameters with high precision through machine learning, in which the impacting factors were divided into static and dynamic groups and used as features to train a learning model for estimation [46]. Elifcan and Rizvan presented a pick-up routing combinatorial problem with a three-dimensional loading constraint, clustered backhauls at the operational level, and trained loading at the tactical level for an intermodal transportation network. It was solved by the combination of mathematical models, genetic algorithm, and machine-learning algorithm [47]. Dai et al. proposed a sub-bus based on the mobile data that contained the 
passenger flow forecasting and dynamic route planning of the stations [48]. Xu et al. came up with a new data-driven method integrating data mining models and facility location models by integrating with customer behavior data analysis, to optimize collection and delivery points for online retailers [49]. Specific to CTHS, Andreas et al. demonstrated the feasibility of deep learning approach by implementing it as part of a terminal productivity cockpit prototype that provided decision support to terminal operators for proactive process adaptation [50]. Zhang et al. put forward novel machine-learning-driven algorithms, which integrated optimization methods and machine-learning techniques, to solve the container relocation problem [51]. Hottung et al. proposed a new method called deep learning heuristic tree search that used deep neural networks (DNN) to learn solution strategies and lower bounds customized to the container pre-marshalling problem solely through analyzing existing or near optimal solutions to container premarshalling problem instances [52].

From the foregoing, it is concluded that the machine learning and deep learning with artificial neural network (ANN) and DNN provide the new methodology and practice for the scheduling, control, decision, and execution of CLS under the dynamic and uncertain environments. In particular, the prediction function provided by deep learning for the key operational indicators of CLS provides the pivotal planning and scheduling benchmark reference for the operational management and executive control. The planning and scheduling of CTHS involves multitudinous discrete and combinatorial optimization issues, especially as the container terminals move toward intelligent logistics systems. The deep learning is bound to develop greatly in the transformation and upgrading of CTHS. Now, the operational complexity of CTHS is going be discussed in more detail in subsequent sections, and then the corresponding solution by the combination of computational logistics and deep learning is brought forward in an attempt to improve the container capacity, response time and service efficiency of CTHS.

\section{Container Terminal Handling Systems}

3.1. Overview of Container Terminal Logistics Services. The container terminal is a buffer pool for container switching modes of transportation and also an efficient distribution center. It is the multimodal transport and comprehensive logistics hub of global supply chain network. Judging from the specific practice, it is essentially a large critical infrastructure of warehousing in the process of logistics service. CTHS are the typical CLS, and are also the discrete event dynamic systems, distributed heterogeneous control systems, and hierarchical parallel and reconfigurable batch processing systems from the various abstract perspectives by computational logistics [53]. CTHS are the most typical representatives of pivotal logistics hubs (PLH), and it has been a hot and difficult subject in the field of CLS.

CTHS are mainly composed of infrastructure, facilities, and equipment (IFE) of terminal quay side, the terminal storage yard, and the IFE of land side besides the auxiliary pilotage IFE, such as navigation marks and tugboats. All movements and functions of CTHS focus on the given container liner set, and the berth is the most valuable productive resource. Therefore, the berthing, unberthing, and handling at quay side are the most important parts of the working of CTHS. Meanwhile, the running of the yard is the most complicated piece in CTHS because it not only responds to the planning and scheduling instructions of quay side and land side simultaneously but also processes the import containers, export containers, and containers for transshipment synchronously. In addition, the IFE of land side is in charge of the overland container collection and distribution that takes up a significant portion of the container throughput, especially in China.

\subsection{Container Terminal Logistics Tactical Operational} Complexity. As a typical representative of PLH and CLS, CTHS possesses the distinguishing features of PDN-CCC, especially for operational complexity. The PSAC for single phrase at container terminals is provided with the distinct characters of NPC [54-56]. As a result, the PSAC solutions whether to the single resource allocation or to the integrated scheduling both are of the evident qualities of strong timeliness and low flexibility. Therefore, it is essential to give a preliminary sketch of the container terminal logistics tactical operational complexity (CTL-TOC).

According to the nature of computation, the handling, stacking, and transportation procedures at container terminals can be abstracted as the container terminal-oriented logistics generalized computation (CTO-LGC) that occurs in the physical world [57]. The generalization and unification of computation is one of the fundamental principles of computation logistics that is deeply rooted in computational thinking [58]. The CTL-TOC can be abstracted and mapped into the control, execution, and feedback of container-terminal-oriented logistics generalized computation (CEFCTO-LGC), and then it may achieve the solution by computational logistics. Judging from the specific practice, the CEF-CTO-LGC is primarily concerned with a series of scheduling and executive decision problems, which have been demonstrated by Figure 1 .

Obviously, the CEF-CTO-LGC is provided with high PDN-CCC even without considering multimodal transportation. Each term in CEF-CTO-LGC is the problem of NPC; moreover, there are the strong, complicated, and tangled correlation, and coupling and cascade effects among them. As a matter of fact, CTL-TOC can be abstracted and translated into an NPC problem network for logistics computation decision (NPN-LCD). The CEF-CTO-LGC lies in and runs through the NPN-LCD. At the same time, the LHV affects the planning and scheduling of almost every problem in NPN-LCD. As a result, we take the LHV forecasting as an example to illustrate the collaborative decision-making and combined application of computational logistics and deep learning.

The distinguishing features of CTO-LGC are the root causes of high CTL-TOC, and those include hierarchy, 


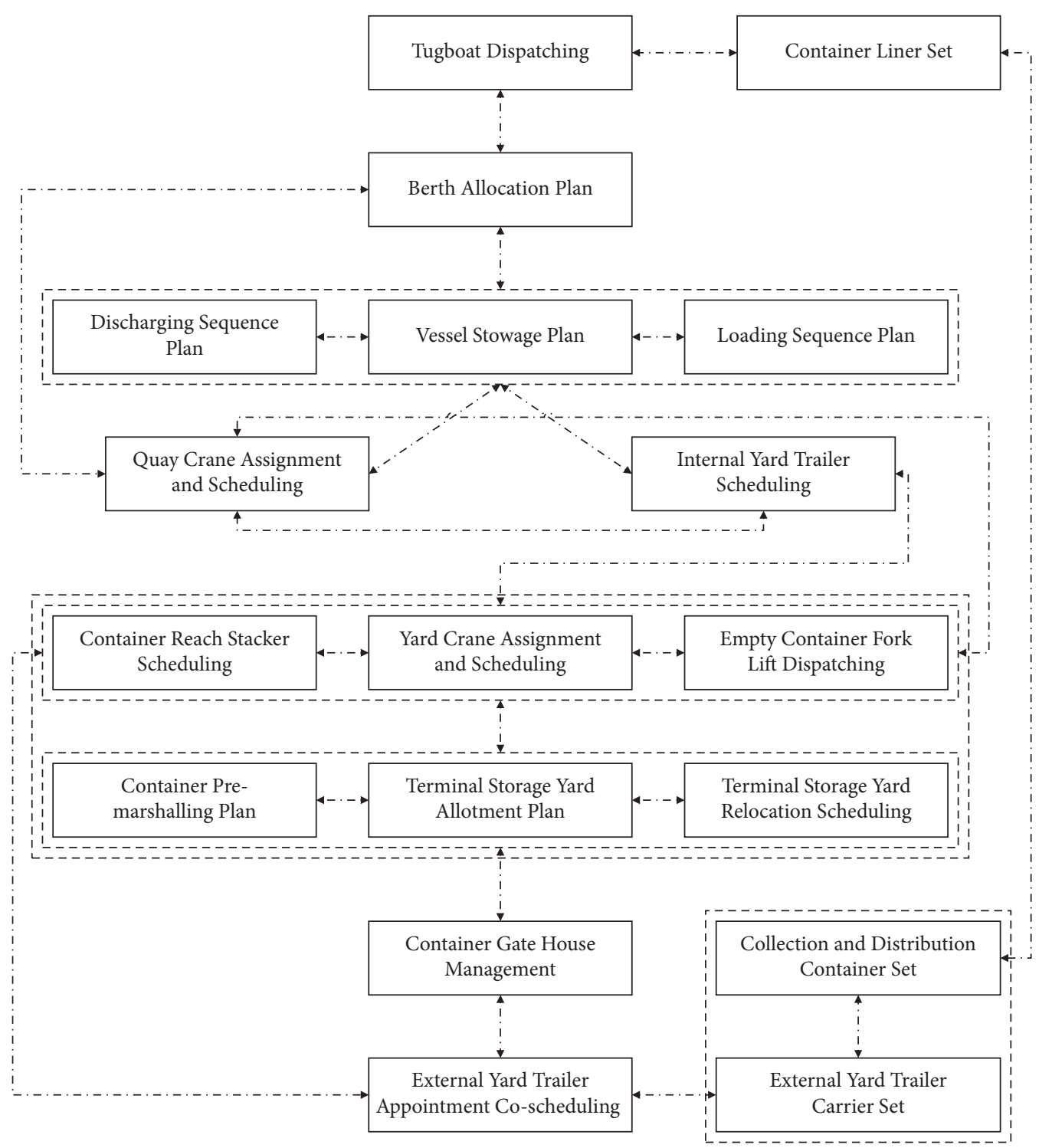

FIGURE 1: Container terminal logistics tactical operational complexity and intrinsic coupling sketch.

dynamics, distributivity, parallelism, locality, affinity, coupling, heterogeneity, reconfigurability, being context-sensitive and goal-oriented. In addition, the indeterminacy, dynamics, and perturbation in CEF-CTO-LGC and service environments exacerbate the CTL-TOC. In a word, the PSAC of container terminal is a typical multi-objective, multistage, multi-constraint soft real-time compound combinatorial optimization problem under uncertain environments. From a generalization point of view, the CTLTOC can be regarded as the entropy perspective abstraction of operational complexity for CTHS to some extent. Apparently, the running entropy of CTHS is going to continue to mount once the treatments of CTL-TOC are adverse.

\subsection{Container-Terminal-Oriented Computational Logistics.} After a decade of exploration and experiment, the definition of computational logistics is preliminarily proposed by Bin Li on the 54th IEEE Conference on Decision and Control
(CDC 2015) [58], which is the problem-oriented exploration, specific application, and industry practice of computational thinking for the CLS technically.

Computational logistics provides a feasible, exercisable, reliable, and robust decision methodology and engineering solution for CLS in the physical world, especially for the public logistics hubs at all levels in global supply chain networks. The container terminal is the very large-scale public logistics infrastructure for the unit logistics; moreover, it has a vital impact on the operation of the global logistics hierarchical and multistage network. As a result, the CTHS has been the typical application to demonstrate the ideology and philosophy of computational logistics. The computational logistics tries to overcome the CTL-TOC by the combination of computational thinking, theory of computation, computational lens, and great principles of computing, which are abbreviated to 4CTTLP [59]. It is distinct from the classical research methods for CTHS. In the meantime, the emergence and development of machine 
learning provides the wing plane of the computational logistics, especially for the booming deep learning in machine learning [60].

After the computational logistics establishes a sound, solid, agile, and robust abstraction and automation foundation of CTHS, the deep learning is supposed to provide the intelligent decision support engine to conquer the NPNLCD of CTL-TOC, at least in part. Now, we take the prediction of container terminal LHV as an example to expatiate on the problem-oriented deep learning model by lightweight, hybrid, flexible, tailorable, scalable, self-organization, self-adaptation, and self-evolution ANN and DNN within the conceptual framework of computational logistics.

\section{Problem-Oriented Deep Learning with Adaptive Neural Networks Computation}

4.1. Problem-Oriented Exploration and Learning of Computational Logistics. The computational logistics is a typical problem-oriented theory, methodology, solution, and practice for CLS by abstraction, mechanization, automation, generalization, unification, transferring, and customization of theory of computation and computing principles because it originates from computational thinking and computational lens inherently. The widespread adoptions of cyberphysical systems and digital twin generate and record practical and massive running process data of CTHS. Those are not only the container terminal logistics service mirror images but also contain a lot of beneficial and adverse logistics service patterns and scheduling decision laws. They are urgently needed for terminal PSAC decisions. However, the 4CTTLP of computational logistics do not contain the relevant theories and methods.

The deep learning provides the means and methods for the above objective as the problem-oriented learning infrastructural accessory package of computational logistics. Since 2006, the deep learning has gradually become prosperous because of the combined effect of some key technology breakthrough and practices. The first and foremost is the significant advances in the design of ANN structures and training strategies, especially for the emergency and boom of DNN. The second is the mass deployed cyber-physical systems and smart phone devices that provide means and methods for the acquisition and utilization of big data for the diverse fields and scenarios. The last point is the fast development of high-performance parallel computing systems, such as graphics processing units (GPU) and general-purpose graphics processing units [61].

For the last fifteen years or so, deep learning has been widely used in the multiple typical domains. Nevertheless, the application of deep learning on scheduling and decisionmaking in the field of production and logistics has been very rare, especially at the tactical and executive level. By computational logistics, the given operational problem for the CLS is carried out by the abstraction, automation, and computational mechanization, and the deep learning is all about a problem-oriented supplementary to implement pattern recognition, self-learning exploration, and selfadaptive improvement. Consequently, the combination of computational logistics and deep learning is supposed to provide a feasible, sound, agile, and efficient methodology, solution, and practice for CLS. In fact, we have already made a preliminary attempt at that in our previous discussion $[62,63]$.

\subsection{Problem-Oriented Lightweight Adaptive Deep Learning} Model for Liner Handling Volume. According to computational logistics, the CTHS can be abstracted as the hierarchical, parallel, heterogeneous, and reconfigurable CTOLGC physical dedicated automatic systems [64]. Meanwhile, the CTO-LGC is the ultimate implementation of container terminal logistics abstraction, mechanization, and automation, and it is the core foundation of the generalized, unified, and quantitative modelling for the execution and decision of CTHS as well. The container terminal is the PLH, and land-and-water coordinated transportation router essentially; moreover, the container liners and the affiliated container set are the central service objects of CTHS. In particular, the container collection is the ultimate execution targets of the mechanization and automation of CTO-LGC.

On the condition that the visiting liners are abstracted as the physical job set for CTHS by computational logistics, the $\mathrm{LHV}$ is similar to the program data volume in the main memory, which is the central indicator of the CTO-LGC throughput and reliability. The LHV is critical and crucial for the accomplishment and execution of CTO-LGC. Furthermore, the LHV is the prerequisite conditions and an essential foundation for the PSAC of CTHS, which is of common concern to both terminals and carriers. In a word, the LHV is a principal line of NPN-LCD, especially for the tactical and execution levels. Hence, we take the evaluation and prediction of LHV as an example to illustrate the application and exploration of the combination of computational logistics and deep learning. Given the importance and volatility of LHV, we initiate a discussion on the LHV prediction, which is intended to better plan and drive the implementation, operation, execution, and tuning of CTHS. We design and implement FEB-LCR-ACM to predict the LHV whose computing architecture is demonstrated by Figure 2.

Revolving around the indicators of LHV, we extract the most relevant performance metrics in the CTO-LGC log, which is the fundamental basis of feature engineering for LHV forecasting. Then, the feature vector of LHV forecasting (FVLF) is given a clear definition whose number of attributes is around 20 according to terminal operational records. Obviously, the label of forecasting is just about LHV itself. Both construct the FVLF raw petroleum dataset for deep learning engine. Meanwhile, because of the operational characteristics of CTHS, the CTO-LGC log set is so small for the deep learning. Usually, there are only thousands of records in the running log for five years or more even for large-scale container terminals. As a result, it is very difficult for traditional machine learning and deep learning to predict LHV.

It is supposed to pull through the bottleneck that the combination of computational logistics and deep learning 


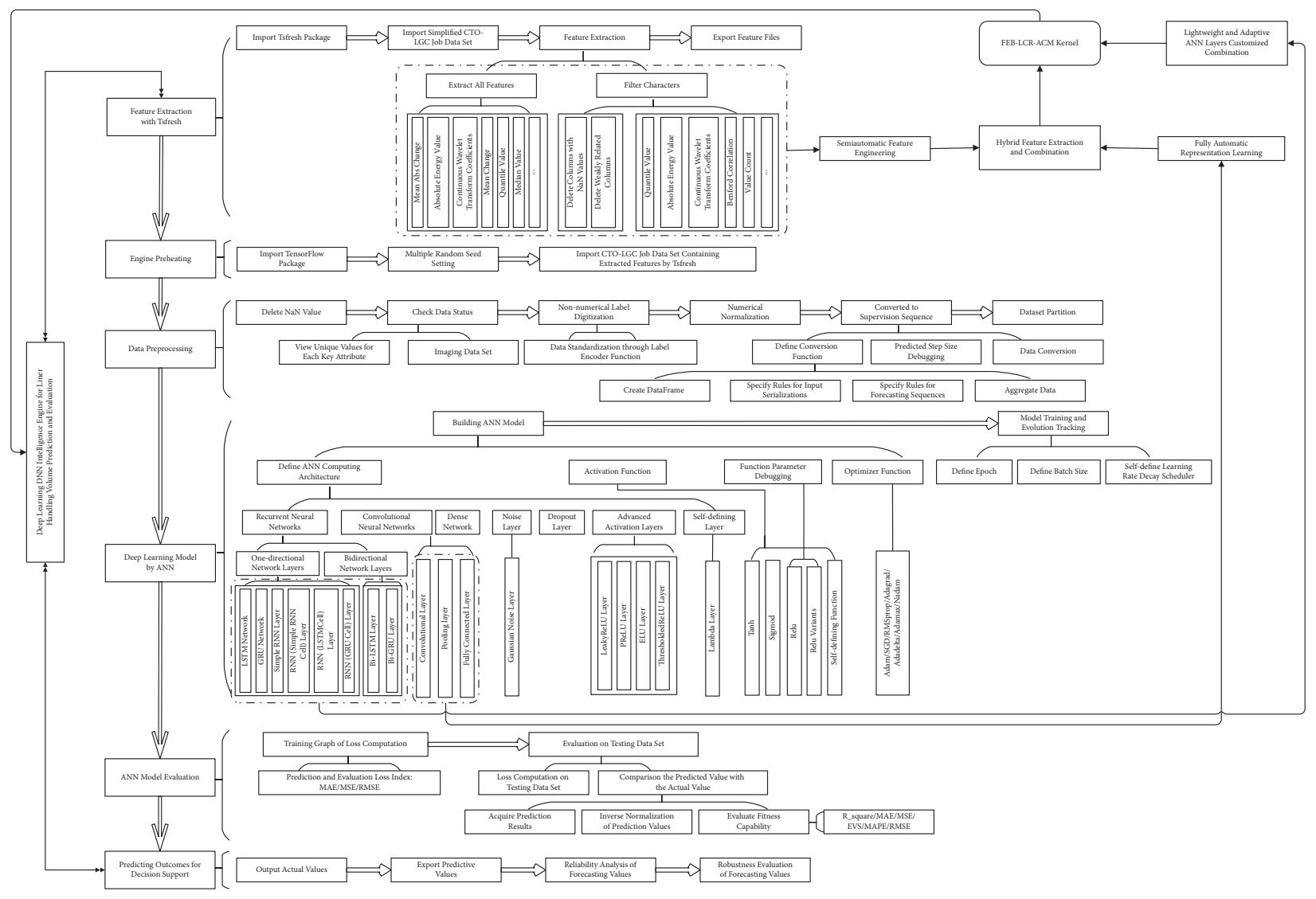

Figure 2: Problem-oriented lightweight adaptive deep learning model for LHV.

presents with a more comprehensive abstraction, automation, intelligence, self-organizing, self-learning, and selfadaptive solution and practice for CTHS. The FEB-LCRACM is just about the consequence of the combined practical application of the above two. The FEB-LCR-ACM for LHV forecasting mainly consists of six components that execute sequentially, namely, feature extraction, deep learning engine preheating, operational mirror data preprocessing, deep learning model, ANN model evaluation, and LHV prediction outcome for intelligent decision support, respectively. The two core components are feature extraction and deep learning model. The two key components showed synergetic effect with each other, and are described below.

For one thing, the feature extraction of CTO-LGC running $\log$ is implemented and performed by a Python package that is time series feature extraction on the basis of scalable hypothesis tests named as tsfresh [65], and the extracted features with tsfresh has been used for multiple types of tasks, for instance, classification, compression, forecasting, detection, recognition, and diagnosis [66-70]. For PSAC, the tsfresh package plays an important role in the deep learning data preparation process. Whether from the number of attributes or from the quantity of records, the CTO-LGC dataset is too small. The tsfresh makes dataset feature mining and feature screening to be expanded with key attributes. Finally, the filtered features are going to be incorporated into the CTO-LGC running log; moreover, the accessorial characteristic of computing indicators is more than the primitive attributes of the terminal log. The above two generate the ultimate data fuel of the deep learning engine to achieve the LHV forecasting. As a matter of fact, the feature exaction by tsfresh is just about the dimensionality raising for the primitive CTO-LGC running log by pattern recognition and shallow learning in essence.

For another, the design and realization of the deep learning model is a typical ANN computing architecture that covers RNN, CNN, dense network, dropout layer, noise layer, advanced activation layer, self-defining layers, and so on. The RNN is further subdivided into four classes: LSTM, gated recurrent unit (GRU), bidirectional LSTM, and bidirectional GRU. The CNN further mines and extracts the characteristics of the data fuel, and it is intended to improve the combustion efficiency of data fuel to implement mapping and forecasting. In consequence, the integration and synergy between RNN and CNN are the key to dramatically increasing the performance of the ANN model. In the meantime, based on a large number of computational experiments, the depth of ANN in FEB-LCR-ACM usually is approximately between four and nine for CTHS, whose computing architecture is less than ten layers in the vast majority of cases. As a result, the FEB-LCR-ACM is a typical lightweight deep learning computing architecture. It is evident that the FEB-LCR-ACM is tailorable, configurable, tunable, and customized depending upon the particular terminals and conditions, which is crucial and critical for the 
rationality, efficiency, agility, flexibility, portability, and robustness of FEB-LCR-ACM.

\subsection{Deep Learning Kernel of Liner Handling Volume} Forecasting. As previously mentioned, the FEB-LCR-ACM must be self-defined and tuned for the given CTHS due to the diversity of the various container terminals. However, for any container terminal, the deep learning kernels of LHV forecasting is relatively stable, exercisable, and workable, which are explained in further detail below.

Above all, the CTO-LGC log dataset around LHV is of outstanding features. The LHV fluctuates sharply over a wide range between single digit and five digits for the whole set of calling container ships. Moreover, in most of the target datasets predicted by ANN, the time series increases uniformly [71, 72]. For all appearances, the liners call at the terminal at irregular intervals, which makes the LHV forecasting even more difficult. In addition, the LHV log at the container terminal must be a small dataset, and it only covers thousands or tens of thousands of records at the most because of the terminal operation features, which is far from big data. Furthermore, the liner routes at container terminals are often drastically adjusted, and even the ships in operation for the specific routes are replaced from time to time according to the rapidly changing economy. However, compared with the liner berthing time (LBT) at container terminals that fluctuates within a range between one hour and 36 hours in most situations [60], the fluctuation degree of the LHV is more than 100 times that of the LBT, and the statistical pattern of the LHV is even less obvious. Hence, we focus on the feature extraction of CTO-LGC running log surrounded by the topic of liners' calling and handling in FEB-LCR-ACM.

Secondly, after the feature extraction by tsfresh, the computing process of CTO-LGC running log dataset before deep learning is also crucial and critical to the performance of FEB-LCR-ACM, which includes numeralization, normalization, supervised serialization, and dataset partitioning. The first three of these are particularly specialized and custom-made for LHV forecasting. Then, the ultimate data frame is beginning to take shape to launch and run the deep learning engine efficiently to explore the intrinsic modes and the law of development for LHV. It is fundamental for the application of deep learning for the PSAC of CTHS. Specific to the LHV forecasting, the numeralization, normalization, and supervised serialization of CTO-LGC log three form a three-dimensional and mutually supportive data frame generation mechanism. Given that there are usually multiple data types in the original dataset of CTO-LGC, the numeralization of CTO-LGC running $\log$ is a necessary and mandatory requirement. The primary task of numeralization is to transform nonnumerical attributes into numerical feature components, such as carrier, ship routing, and voyage. Furthermore, the numerical code cannot introduce new features into the dataset; otherwise, the FEB-LCR-ACM may try to find a law that does not exist in CTHS, resulting in overfitting. In the meantime, because the operation of CTHS has great dynamics, randomness, nonlinearity, and uncertainty, it can easily lead to a state of overfitting while applying FEB-LCR-ACM to the CTO-LGC log. As a result, the normalization is of great importance to avoid overfitting and underfitting. In addition, the supervised serialization plays an important role for the generation of data frame by defining conversion function, specifying rules for input, specifying rules for forecasting sequences, and aggregating data.

Thirdly, the combination of RNN and CNN is common in deep learning. However, it is rarely applied to production management and scheduling decision, especially for CLS. Through our attempt and exploration on the LHV forecasting, it has been proved essentially to be a feasible, valid, and efficient approach to the intelligent decision support for PSAC. The tsfresh is a kind of semiautomated feature extraction means, and the $\mathrm{CNN}$ is a purely automatic feature extraction approach. Both get the utmost out of the original dataset to help the RNN to implement and improve the forecasting performance. In other words, the CNN and tsfresh make joint efforts to design and implement the feature extraction of CTO-LGC running log to improve the forecasting performance of the FEB-LCR-ACM. Fundamentally speaking, the tsfresh pays close attention to the statistical characteristics of CTO-LGC running log selectively, and the CNN places emphasis on the spatial feature mapping. Subsequently, the RNN focused on the time series prediction of LHV on the basis of the feature vectors set processed by feature engineering with the combination of tsfresh and CNN.

Lastly, the RNN has a large number of self-defined components that includes multiple activation functions, lots of hyperparameters, multifarious optimizers, and so on. The combination of unidirectional LSTM and GRU, bidirectional LSTM and GRU, cell LSTM and GRU, noise layers, advanced activation layers, and self-defined layers constitute the main body of the predictive computing engine together with the classical dense network layer and dropout layer. The composition and structure make the FEB-LCR-ACM to be endowed with good flexibility, wide adaptability, and real portability.

Thereupon, the integration of the three essential core elements, which are no other than the feature extraction packages of tsfresh, RNN and CNN, constitute a three-dimensional lightweight exploration computing architecture for the operation of CTHS. Thereinto, the tsfresh implements a semiautomatic feature engineering, and the $\mathrm{CNN}$ achieves a fully automatic representation learning. As a result, both construct a hybrid feature extraction and combination for deep learning model. The RNN fulfills a lightweight and adaptive ANN layers customized combination for the given CTHS. The above three establish an agile, efficient, and robust kernel for the FEB-LCR-ACM. The kernel can achieve excellent performance even in the face of the extremely complicated situations, which fully explains the feasibility, applicability, and practicability of the FEB-LCR-ACM. It can provide a better managerial insight into the uncertainty and volatility in future demands for CTHS, and is critical for constructing an agile, efficient, and robust physical logistics services as well. 


\section{Experiments, Results, and Analysis}

5.1. Case Scenario and Loading Job Set Evaluation. A typical, large-scale container terminal in China is chosen as the research target to probe into the FEB-LCR-ACM. The annual container throughput of the terminal is about 1.8 million twenty feet equivalent units (TEUs), which is distinguished from container units. The terminal is equipped with five berths and 12 QCs along the coastwise wharf. More than $80 \%$ of the routes are domestic container liners, and the rest are international container shipping ones. This is a very typical regional container terminal hub in China.

Because the domestic routes liner takes up the main body of calling ships, we select the corresponding liner calling, berthing, and handling log as the loading task set of the CTO-LGC to design and implement the LHV forecasting. Above all, we intercept the CTO-LGC log data for six years to make the evaluation of $\mathrm{LHV}$, which is an important basis of further prediction. There are 13,873 container vessels for the domestic trade to visit the terminal during this period. It is a physically demanding job set for CTO-LGC for the six years to execute the quantitative random job testing for CTHS [73]. The preliminary sketch of LHV can be illustrated by Figure 3 and Table 1, and two distinct features are shown in both. For one thing, it is easily concluded that the LHV is highly volatile and has randomness relative to LBT [62] because the domestic liners are very diverse for different routes. For another, one feature stands out. It is that the great majority of LHV are within 1000 TEUs, which comes up to 12897 items and accounts for over 92 percent of all records. The LHV records within 2000 TEUs reach up to 98.57 percent. To some extent, the rest of the 198 ones exert strong stochastic disturbance for the prediction of LHV, However, those are the key and critical CTO-LGC tasks with high priorities that cannot be ignored in practice.

5.2. Machine Learning for Liner Handling Volume Analysis and Prediction. We apply the k-means algorithm to design and implement the cluster analysis of the CTO-LGC log, in order to further evaluate the running characteristics of CTHS for this case. The selected cluster variables include service route encoding, shipping company encoding, liner berthing time, the separate volume of imported containers by three specifications (including 20, 40, and 45 feet), the total import volume by container units, the total import volume by TEUs, the respective volume of exported containers by three specifications (including 25, 40, and 45 feet), the total export volume by container units, the total quantity of export TEUs, the total handling volume container units, the total amount of loading and discharging TEUs, shifting quantity of hatch covers, and so on. The above elements all are of great importance for the NPN-LCD for any of the CTO-LGC stage or their integration. Nevertheless, those are considerably different from the feature vector for the prediction of LBT [60]. At the same time, the CTO-LGC log set is over 10,000 pieces of records, which is much more than the dataset for the discussion of LBT $[60,62]$.
The final cluster centers of the CTO-LGC log are shown in Table 2. The CTO-LGC log is divided into five categories, and the number of cases in each category is $177,7026,543$, 4828 , and 1299, respectively. It shows that the differences of LHV among liners are still significant even if only for domestic trade routes because of the evident distance among cluster centers. In the meantime, the difficulty level of LHV forecasting is interpreted clearly from another perspective.

Subsequently, we discuss the LHV forecasting by the support vector machine (SVM), which is a classical machine learning algorithm based on statistical learning theory $[74,75]$. The advantage of SVM is mainly reflected in solving the linear inseparable and regression prediction problem by introducing the special kernel function.

In view of the fact that the prediction of $L H V$ is a representative regression problem, the CTO-LGC $\log$ is nonlinear in nature from the insight into the mechanized execution, automation control, and combinatorial optimization; moreover, the sample size of the dataset is much larger than the number of attributes in the feature vector. Consequently, the SVM model with Gaussian kernel function is used for LHV forecasting. Besides, the box constraint parameter value is 250 , the epsilon parameter value is 25 , and the kernel scale parameter value is 2 , and the data are standardized as well. Based on the CTO-LGC log dataset of six years, we execute the LHV forecasting for 350 ships by the trained Gaussian SVM model whose experimental results are shown in Figure 4.

The Gaussian SVM model has a medium performance, especially in the high value region of LHV, because the LHV has been jittery over a large range. Obviously, the classical machine-learning algorithm has been unable to meet the requirements of PSAC for CTO-LGC. Now, the FEB-LCRACM is introduced into the LHV forecasting to execute the problem-oriented exploration by the combination of computational logistics and deep learning.

5.3. Deep Learning for Liner Handling Volume Forecasting. The FEB-LCR-ACM is a lightweight deep learning model, and it does not require much hardware. It is constructed, implemented, executed, and debugged based on the single GPU of the NVIDIA GeForce GTX 1660 Ti. The GPU compute capability of compute unified device architecture is only 7.5, and the video memory is just $6 \mathrm{~GB}$. The time that the FEB-LCR-ACM runs once usually is less than two minutes in this case. There is no doubt that it provides a good prerequisite for the intelligent decision support for CTOLGC at the tactical and executive level within the given soft real-time constraint conditions in industry practice.

Based on a large number of computational experiments that is designed and implemented with the assistance of tsfresh 0.170 , the depth of DNN is usually approximately between four and nine for the multifarious CTHS. In this case, the DNN for LHV prediction is defined, constructed, executed, and debugged by the six-layer architecture, which is implemented by TensorFlow 2.3 for GPU. Meanwhile, the multiple activation functions of deep learning are applied in 


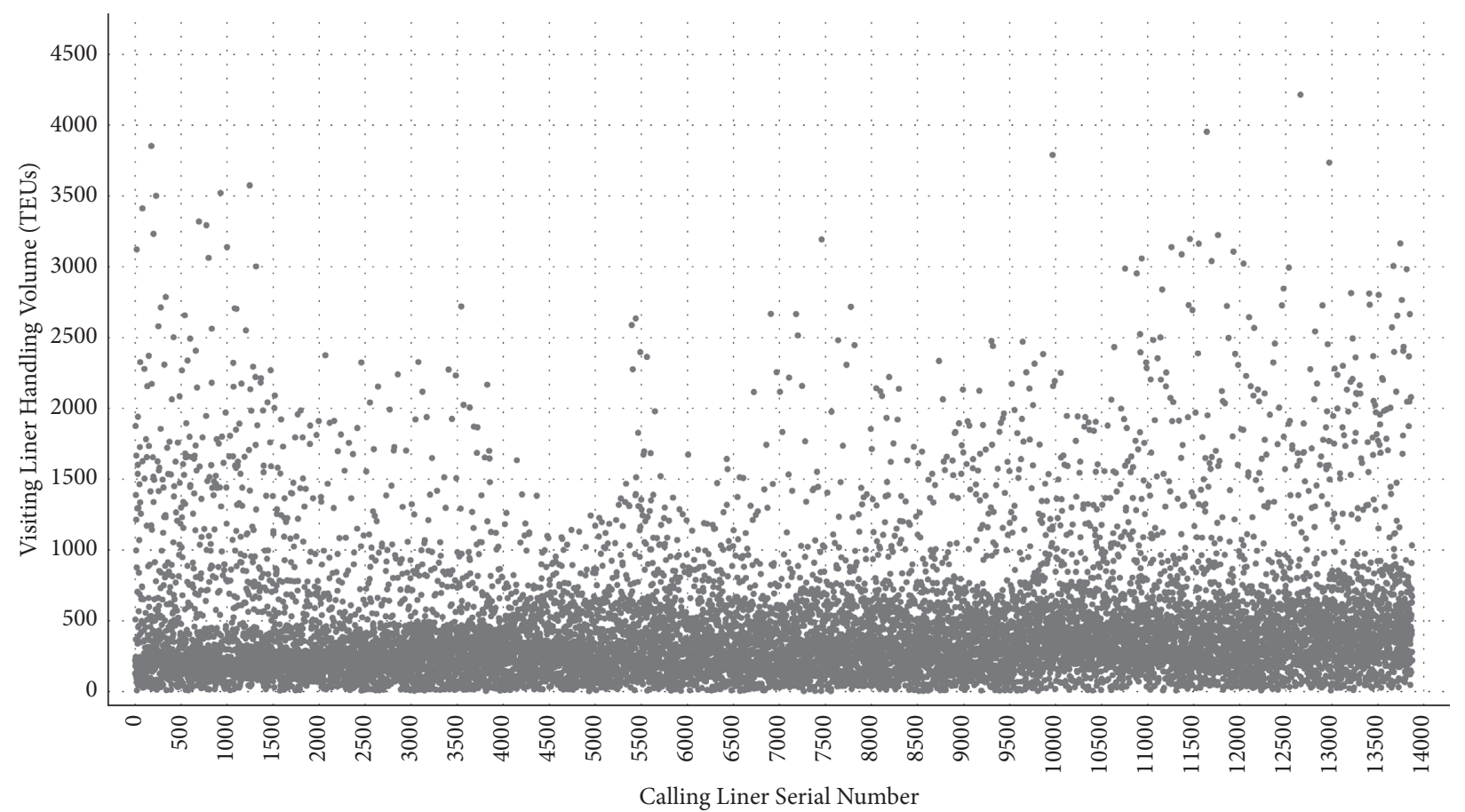

FIgURE 3: Distribution preliminary sketch of calling liners handling volume.

TABLE 1: Distribution characteristics of LHV for domestic trade routes (unit: TEU).

\begin{tabular}{lcccccccc}
\hline Year & $\begin{array}{c}\text { Quantity of } \\
\text { Liners }\end{array}$ & $\begin{array}{c}\text { Minimum of } \\
\text { LHV }\end{array}$ & $\begin{array}{c}\text { Maximum of } \\
\text { LHV }\end{array}$ & $\begin{array}{c}\text { Mean of } \\
\text { LHV }\end{array}$ & $\begin{array}{c}\text { Median of } \\
\text { LHV }\end{array}$ & $\begin{array}{c}\text { Mode of } \\
\text { LHV }\end{array}$ & $\begin{array}{c}\text { Standard deviation } \\
\text { of LHV }\end{array}$ & $\begin{array}{c}\text { Variance of } \\
\text { LHV }\end{array}$ \\
\hline YA & 1558 & 8.0000 & 3853.5000 & 463.7943 & 237.5000 & 150.0000 & 554.1087 & 307036.4432 \\
YB & 2056 & 5.0000 & 2720.0000 & 335.0133 & 246.0000 & 246.0000 & 328.2853 & 107771.2362 \\
YC & 2213 & 2.0000 & 2635.0000 & 359.4288 & 291.0000 & 144.0000 & 297.2632 & 88365.3966 \\
YD & 2647 & 2.0000 & 3193.0000 & 376.1501 & 296.0000 & 186.0000 & 324.3637 & 105211.8082 \\
YE & 2563 & 3.0000 & 3790.0000 & 467.5443 & 373.0000 & 194.0000 & 400.7074 & 160566.4049 \\
YF & 2836 & 7.0000 & 4215.0000 & 521.4703 & 395.0000 & 388.0000 & 496.3402 & 246353.5868 \\
\hline
\end{tabular}

TABLe 2: Final cluster centers of CTO-LGC log dataset with the six years.

\begin{tabular}{|c|c|c|c|c|c|}
\hline Cluster variables & Cluster 1 & Cluster 2 & Cluster 3 & Cluster 4 & Cluster 5 \\
\hline Liner berthing time & 25.552 & 8.049 & 19.985 & 13.076 & 16.546 \\
\hline Import volume of 20 -feet container units & 768.000 & 39.000 & 593.000 & 125.000 & 363.000 \\
\hline Import volume of 40 -feet container units & 155.000 & 23.000 & 116.000 & 36.000 & 60.000 \\
\hline Import volume of 45 -feet container units & 3.000 & 0.000 & 1.000 & 1.000 & 0.000 \\
\hline Total import volume by container units & 926.000 & 62.000 & 710.000 & 161.000 & 423.000 \\
\hline Total import volume by TEUs & 1085.380 & 85.015 & 827.633 & 197.794 & 484.084 \\
\hline Export volume of 20 -feet container units & 1166.000 & 43.000 & 588.000 & 124.000 & 256.000 \\
\hline Export volume of 40 -feet container units & 113.000 & 27.000 & 87.000 & 64.000 & 52.000 \\
\hline Export volume of 45 -feet container units & 2.000 & 1.000 & 1.000 & 1.000 & 0.000 \\
\hline Total export volume by container units & 1281.000 & 71.000 & 676.000 & 189.000 & 308.000 \\
\hline Total export volume by TEUs & 1395.867 & 99.508 & 764.316 & 254.288 & 360.371 \\
\hline Total handling volume by container units & 2207.000 & 133.000 & 1386.000 & 351.000 & 731.000 \\
\hline Total handling volume by TEUs & 2481.247 & 184.523 & 1591.949 & 452.082 & 844.455 \\
\hline Shifting quantity of hatch cover & 47.000 & 11.000 & 36.000 & 19.000 & 23.000 \\
\hline
\end{tabular}

diverse ANN layers, and it includes rectified linear unit, Sigmod, Tanh, etc. Besides, the training epoch is 60, and the batch size is 30 . It is evident that the DNN model is tailorable, configurable, and customized, which is crucial and critical for the efficiency, agility, flexibility, and portability of FEB-LCR-ACM.
The whole CTO-LGC log is divided into two datasets, which is intended to ensure that the FEB-LCR-ACM does not overfit or underfit. One is the CTO-LGC log for five years that includes 11037 items, which is named after LHVIVE. The other is CTO-LGC log for six years that covers 13873 ones, which is named after LHV-SIX. The former is 


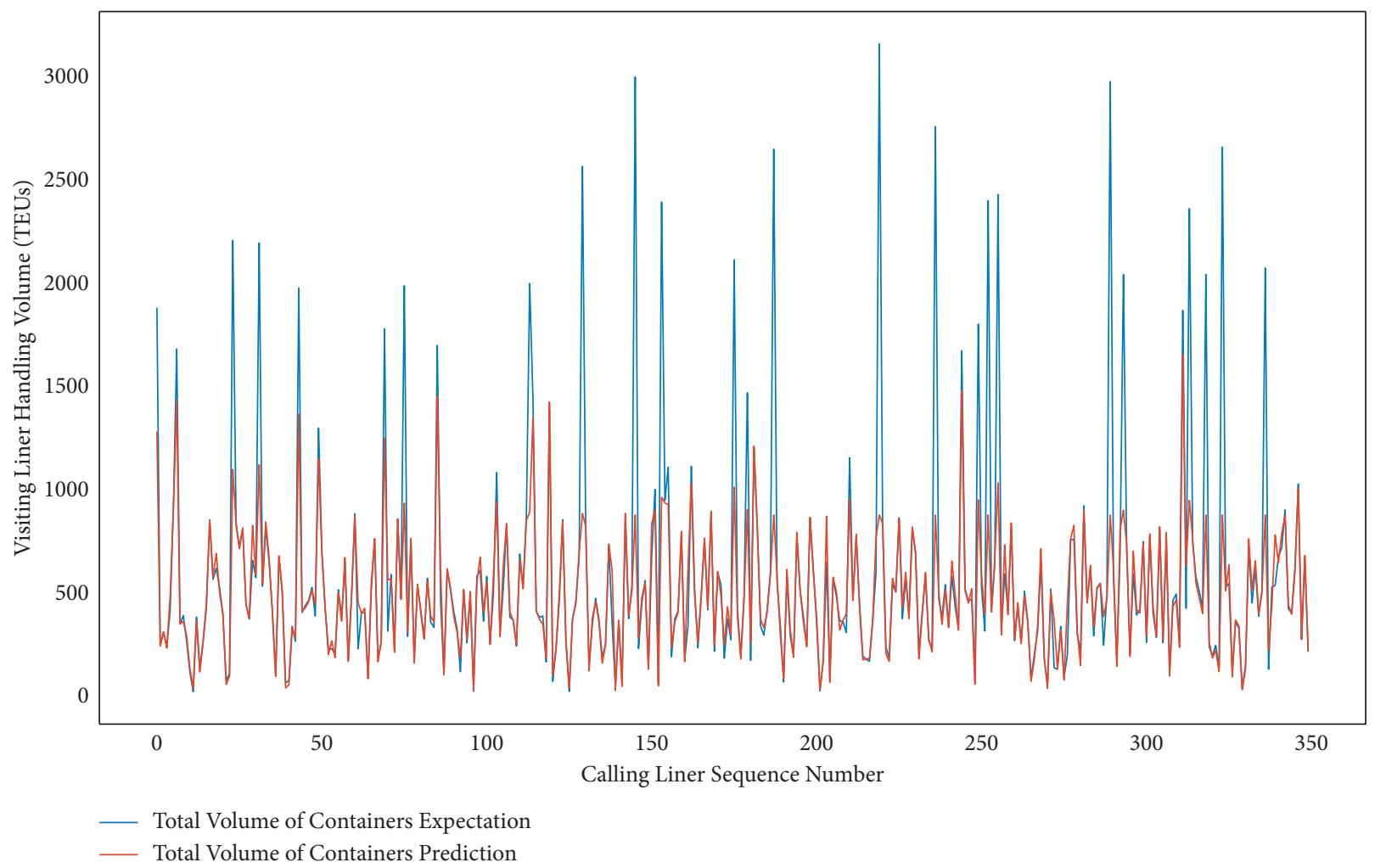

FIgURE 4: A comparison of LHV predicted and true values by the Gaussian SVM model.

segmented into three main sections that are the training set, validation set, and the testing set, and the proportion of the three are $80 \%, 17.282 \%$, and $2.718 \%$, respectively. The latter is also divided into three subsets, and the proportions are $80 \%, 17.477 \%$, and $2.523 \%$, respectively.

It is important to emphasize that the application mode of tsfresh is special processing for the prediction of LHV. The tsfresh package is applied to conduct feature mining with the handling container quantity by TEU as the target variable, and over ten characteristic computing indicators are obtained explicitly. After a number of extraction and comparison experiments, the combination of absolute energy value and Mexican cap can help the DNN learning model to achieve excellent performance. The former is a single indicator, and the latter includes four indicators according to the different parameters. These five feature indicators are computed for an original attribute. The multiple raw properties are going to execute the feature engineering by tsfresh, which include LBT, total import volume by container units, total import volume by TEUs, total export volume by container units, total export volume by TEUs, total handling volume by container units, and total handling volume by TEUs. As a result, a total of 35 indicators are appended to the original dataset by feature engineering.

Consequently, the LHV of 300 ships and 350 liners are going to be forecasted that are about four to five weeks of liners for China's domestic trade requirements. In other words, the FEB-LCR-ACM can make a decision support for the CTO-LGC planning and scheduling of one month or more in this case. We performed 100 times for the two datasets separately with the same random number seed set; moreover, the running of DNN model has excellent reproducibility for the same seed. The typical DNN model training loss curves can be illustrated by Figures 5 and 6 . The contrast between typical LHV forecasting with actual values can be displayed by Figures 7 and 8, respectively. Moreover, the comparison between the mean of LHV forecasting and actual values are demonstrated by Figures 9 and 10 independently.

All provide a reference of LHV by a relatively smooth and reliable pattern with low time and computing costs because of compound feature extraction and lightweight self-adaptive computation, which is widely applicable to various value ranges. Meanwhile, the prediction deviation profiles of LHV are showed by Tables 3 and 4 that present a comprehensive understanding for the performance of the DNN model. All suggest that the FEB-LCR-ACM can acquire a sound and dependable decision reference of LHV using a feasible, reliable, and efficient mode. The FEB-LCRACM demonstrates good follow-up and credibility; furthermore, it is neither overfitting nor underfitting.

Based on Figures 7-and 10, Tables 3 and 4, it is easy to draw the conclusion that the FEB-LCR-ACM supplies the sound and efficient references for intelligent decision support of CTHS. In the case of severe vibration of LHV, the prediction error of LHV within 100 TEUs is close to $80 \%$ based on LHV-IVE, and the prediction error of LHV within 100 TEUs is close to $90 \%$ based on LHV-SIX. That can bring great conveniences for the PSAC of CTHS. 


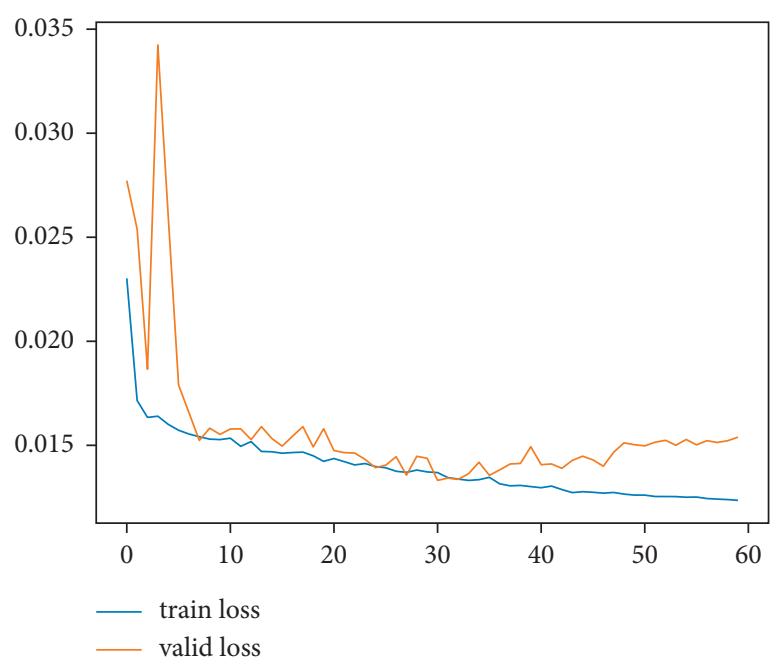

FIgUre 5: A typical FEB-LCR-ACM training loss curve for LHV-IVE.

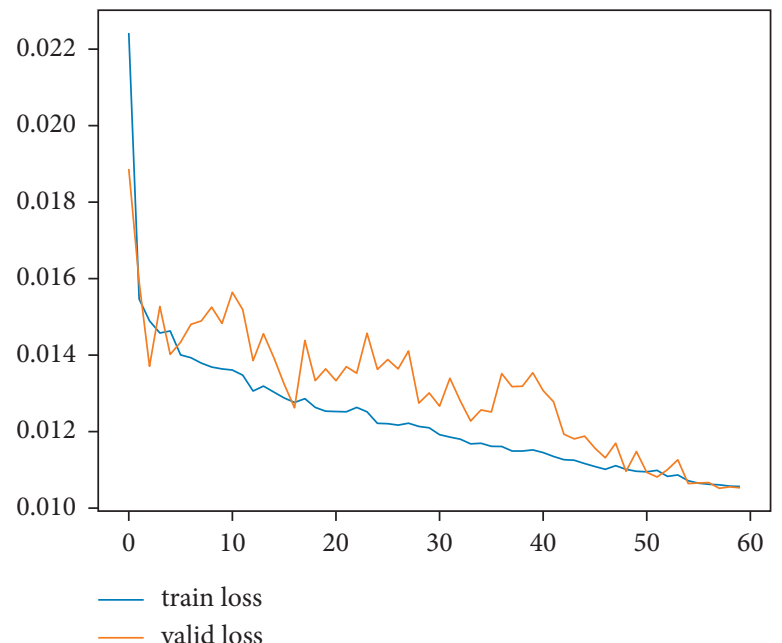

FIgUre 6: A typical FEB-LCR-ACM training loss curve for LHV-SIX.

5.4. Forecasting Performance Evaluation and Analysis. The FEB-LCR-ACM provides a big clue and a favorable baseline to improve the precision, efficiency, and capacity of CTO-LGC. In fact, it shows excellent performance at any stage of deep learning. For one thing, the index of mean absolute error (MAE) is utilized to evaluate evolution and learning effects of DNN model during the phase of training and validation. For the LHV-IVE, at the end of training, the final MAE is between 0.0121 and 0.0126 for 100 experiments, and the average value is 0.0124 . In the validation phase, the final MAE is between 0.0145 and 0.0161 in 100 experiments, and the mean is 0.0152 . With respect to LHV-SIX, at the end of training, the final MAE is between 0.0101 and 0.0110 , and the average value is 0.0106 . In the validation phase, the final MAE is between 0.0099 and 0.0127 , and the mean is 0.0109 . All are close to the theoretical optimal value of MAE.

The MAE, root mean squared error (RMSE), coefficient of determination of R-square, and explained variance score (EVS) jointly construct the core capability dimensions of LHV forecasting performance for the testing phase. The theoretical optimal value of the first two is 0 , and the optimum of the latter two is one. On the one hand, while applying the SVM model with Gaussian kernel function, the MAE is 122.4650 , and the RMSE is 372.1667. The R-square is 0.5420 , and the EVS is 0.5642 . On the other hand, the experimental results of the FEBLCR-ACM are displayed in Tables 5 and 6 under the above evaluation indicators, which has outstanding performance, especially for the latter two. Moreover, the standard deviation and variance of $R$-square and EVS are within an inch of 0 , which shows that the LHV forecasting performance is stable, workable, credible, and excellent. All indicate that the FEB-LCR-ACM provides the prominent predictive behaviors for LHV with a stable and low computing consumption.

5.5. Further Discussion. To sum up, the FEB-LCR-ACM possesses good comprehensive performance. However, there is still much room for improvement on the FEB-LCR-ACM. It 


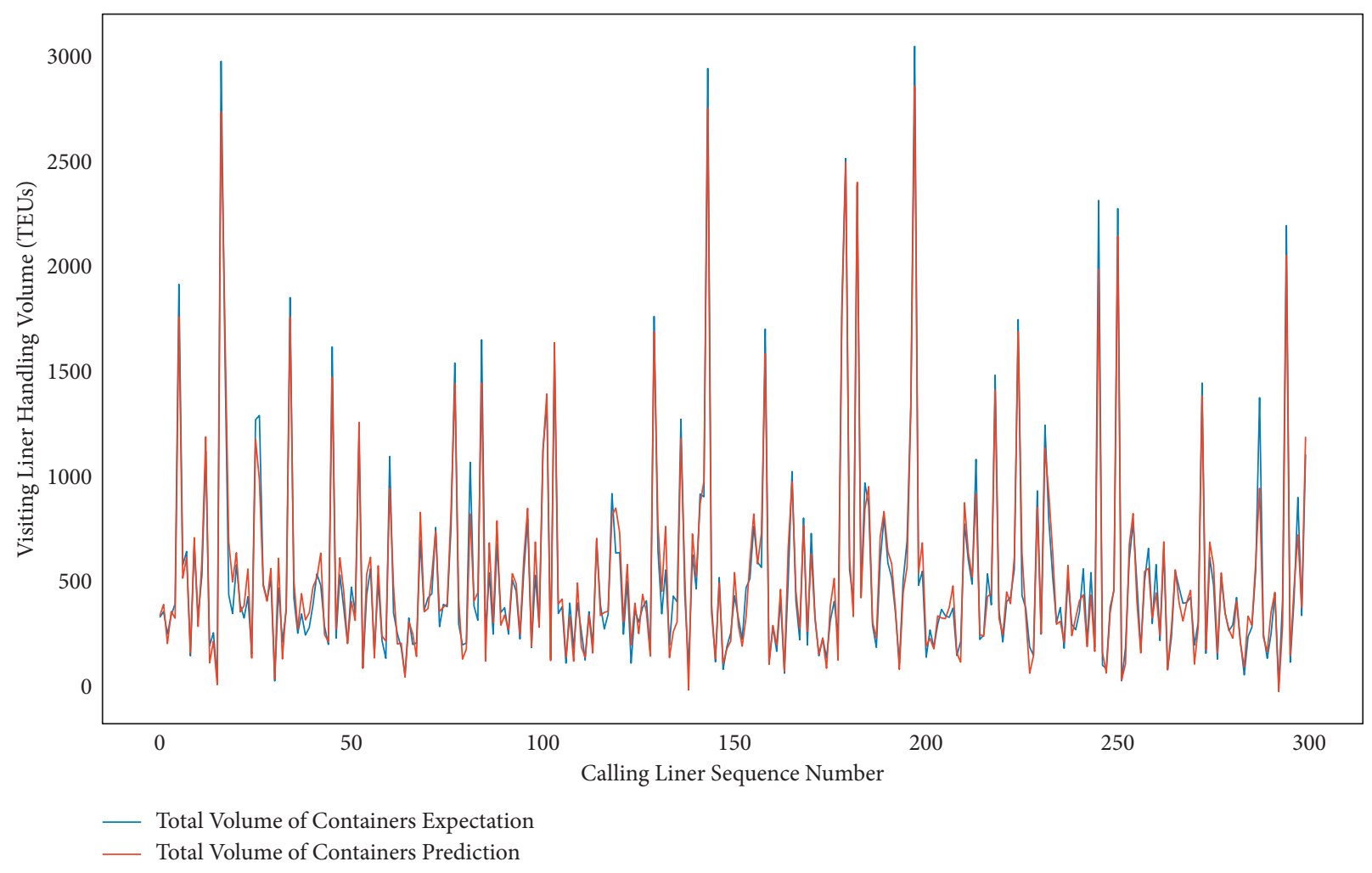

Figure 7: A comparison of predicted values and true values of LHV by LHV-IVE.

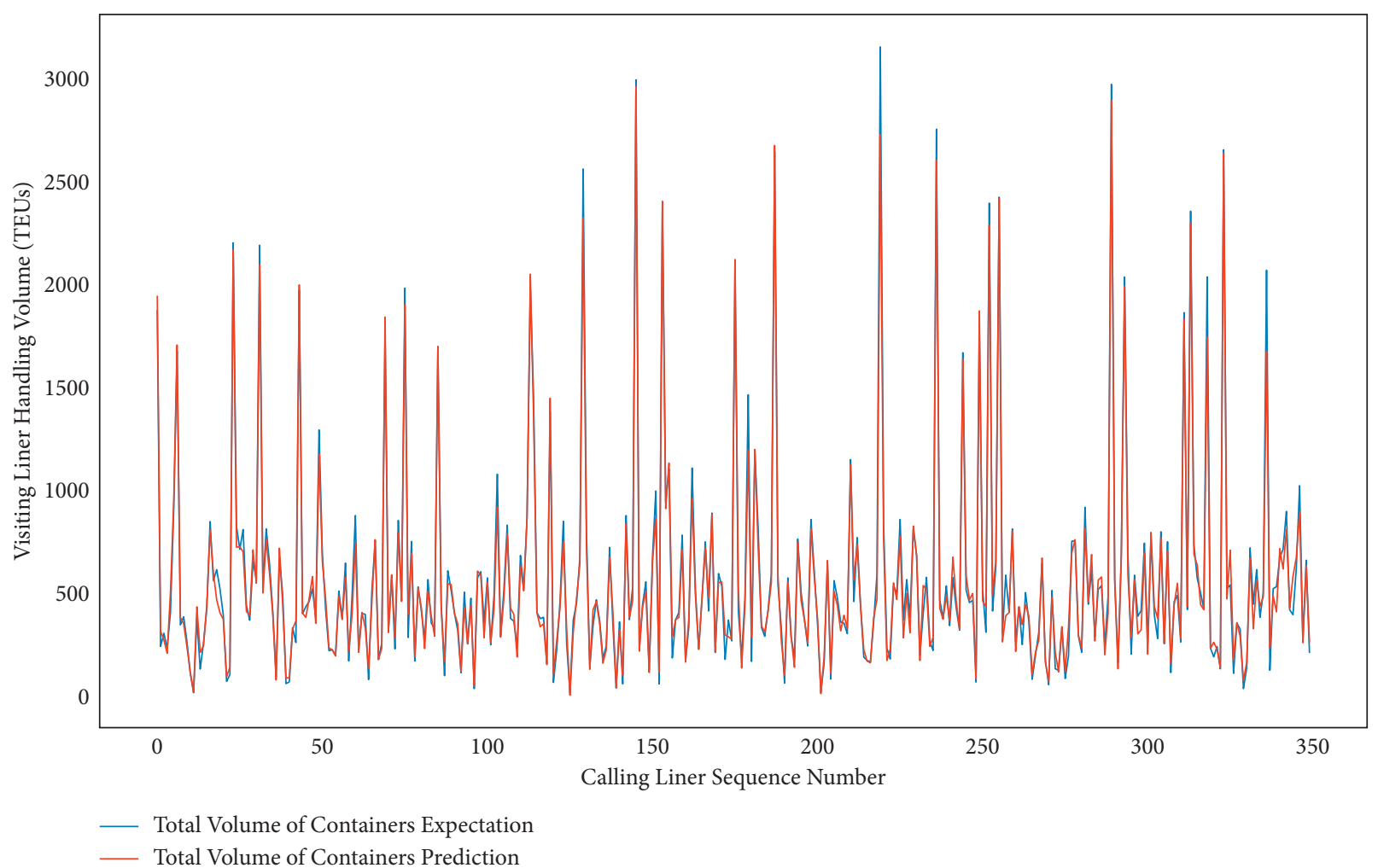

FIgURE 8: A comparison of predicted values and true values of LHV by LHV-SIX. 


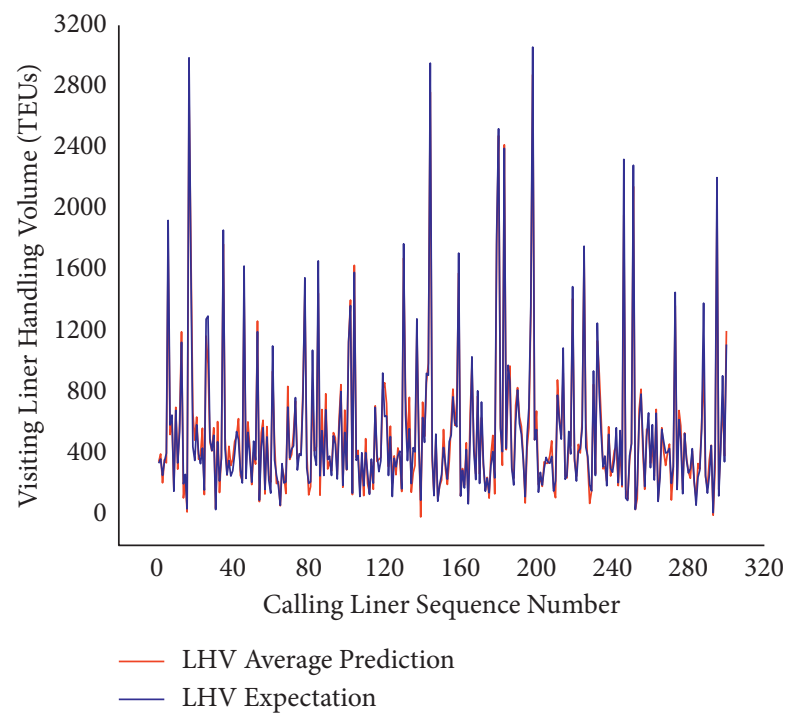

FIGURE 9: A comparison of LHV prediction average results with real values by LHV-IVE.

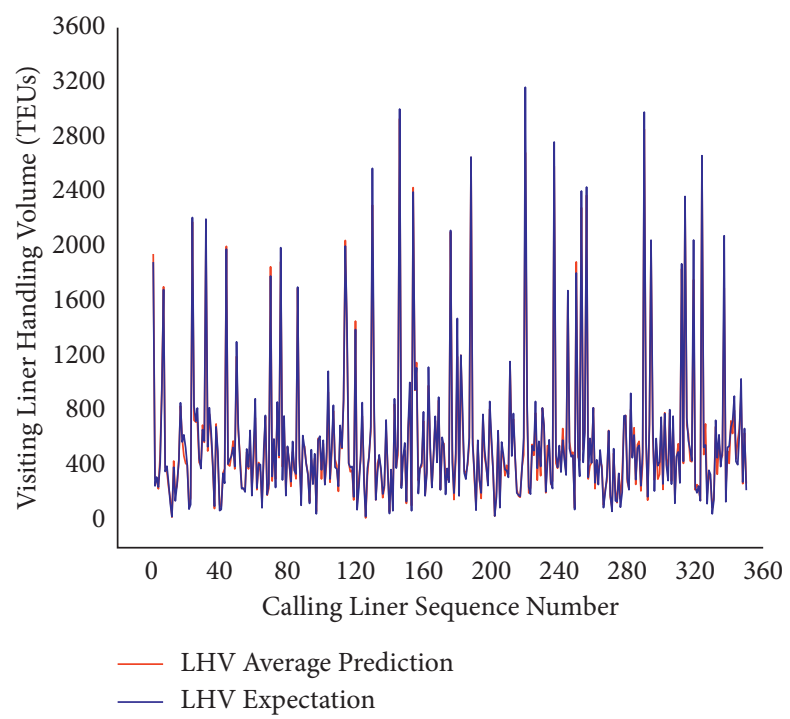

FIGURE 10: A comparison of LHV prediction average results with real values by LHV-SIX.

TABLE 3: Prediction deviation profile of LHV with FEB-LCR-ACM by LHV-IVE (unit: TEU).

\begin{tabular}{|c|c|c|c|c|c|c|c|c|}
\hline $\begin{array}{l}\text { Prediction } \\
\text { deviation of } \\
\text { LHV }\end{array}$ & $\begin{array}{l}\text { Minimum of } \\
\text { liners }\end{array}$ & $\begin{array}{l}\text { Maximum of } \\
\text { liners }\end{array}$ & $\begin{array}{l}\text { Mean of } \\
\text { liners }\end{array}$ & $\begin{array}{l}\text { Median of } \\
\text { liners }\end{array}$ & $\begin{array}{l}\text { Mode of } \\
\text { liners }\end{array}$ & $\begin{array}{c}\text { Standard } \\
\text { deviation of } \\
\text { liners }\end{array}$ & $\begin{array}{l}\text { Variance of } \\
\text { liners }\end{array}$ & Quantitative proportion (\%) \\
\hline$(0,20]$ & 56.0000 & 77.0000 & 64.5800 & 64.5000 & 64.0000 & 3.7926 & 14.3836 & 21.5267 \\
\hline$(20,40]$ & 47.0000 & 67.0000 & 59.0500 & 59.0000 & 57.0000 & 4.2341 & 17.9275 & 19.6833 \\
\hline$(40,60]$ & 38.0000 & 61.0000 & 50.3400 & 51.5000 & 52.0000 & 4.8233 & 23.2644 & 16.7800 \\
\hline$(60,80]$ & 26.0000 & 46.0000 & 36.2400 & 36.0000 & 34.0000 & 3.6664 & 13.4424 & 12.0800 \\
\hline$(80,100]$ & 18.0000 & 36.0000 & 26.3000 & 26.5000 & 27.0000 & 3.9509 & 15.6100 & 8.7667 \\
\hline$(100,120]$ & 15.0000 & 31.0000 & 21.6000 & 22.0000 & 22.0000 & 3.1812 & 10.1200 & 7.2000 \\
\hline$(120,+\infty]$ & 32.0000 & 50.0000 & 41.8900 & 42.0000 & 39.0000 & 3.5381 & 12.5179 & 13.9633 \\
\hline
\end{tabular}

can be inferred from the ANN model training loss curve, especially for valid loss changing curve. One of the worst ANN models validating loss curves for LHV-IVE is shown in Figure 11. Obviously, there are some deficiencies in the validating loss evolutionary process compared with the training loss. Actually, throughout the whole group of 100 experiments, the flaw has always existed, and those are only different in degree, but there is no difference in essence. We extract the optimal 
TABLE 4: Prediction deviation profile of LHV with FEB-LCR-ACM by LHV-SIX (unit: TEU).

\begin{tabular}{lcccccccc}
\hline $\begin{array}{l}\text { Prediction } \\
\text { deviation of } \\
\text { LHV }\end{array}$ & $\begin{array}{c}\text { Minimum of } \\
\text { liners }\end{array}$ & $\begin{array}{c}\text { Maximum of } \\
\text { liners }\end{array}$ & $\begin{array}{c}\text { Mean of } \\
\text { liners }\end{array}$ & $\begin{array}{c}\text { Median of } \\
\text { liners }\end{array}$ & $\begin{array}{c}\text { Mode of } \\
\text { liners }\end{array}$ & $\begin{array}{c}\text { Standard } \\
\text { deviation of } \\
\text { liners }\end{array}$ & $\begin{array}{c}\text { Variance of } \\
\text { liners }\end{array}$ & Quantitative proportion (\%) \\
\hline$(0,20]$ & 86.0000 & 119.0000 & 105.000 & 105.000 & 105.000 & 6.6182 & 43.8000 & 30.0000 \\
$(20,40]$ & 64.0000 & 102.0000 & 82.5300 & 82.0000 & 76.0000 & 8.9638 & 80.3491 & 23.5800 \\
$(40,60]$ & 50.0000 & 75.0000 & 62.3500 & 62.0000 & 61.0000 & 5.4321 & 29.5075 & 17.8143 \\
$(60,80]$ & 26.0000 & 54.0000 & 36.7400 & 36.0000 & 35.0000 & 5.2357 & 27.4124 & 10.4971 \\
$(80,100]$ & 16.0000 & 34.0000 & 24.7500 & 24.0000 & 24.0000 & 3.7320 & 13.9275 & 7.0714 \\
$(100,120]$ & 9.0000 & 28.0000 & 15.4300 & 15.0000 & 16.0000 & 3.8555 & 14.8651 & 4.4086 \\
$(120,+\infty]$ & 17.0000 & 37.0000 & 23.2000 & 23.0000 & 20.0000 & 3.8393 & 14.7400 & 6.6286 \\
\hline
\end{tabular}

TABle 5: Predictive performance evaluation profiles of LHV for the testing phase by LHV-IVE.

\begin{tabular}{lcccccc}
\hline $\begin{array}{l}\text { Evaluation } \\
\text { indicators }\end{array}$ & $\begin{array}{c}\text { Minimum of } \\
\text { indicators }\end{array}$ & $\begin{array}{c}\text { Maximum of } \\
\text { indicators }\end{array}$ & $\begin{array}{c}\text { Mean of } \\
\text { indicators }\end{array}$ & $\begin{array}{c}\text { Median of } \\
\text { indicators }\end{array}$ & $\begin{array}{c}\text { Standard deviation of } \\
\text { indicators }\end{array}$ & Variance of indicators \\
\hline MAE & 62.0850 & 69.6746 & 65.0084 & 64.9422 & 1.4611 & 2.1347 \\
RMSE & 83.9257 & 93.3623 & 87.7590 & 87.5726 & 1.9601 & 3.8421 \\
R-square & 0.9651 & 0.9718 & 0.9692 & 0.9693 & 0.0014 & 0.0000 \\
EVS & 0.9652 & 0.9719 & 0.9692 & 0.9694 & 0.0014 & 0.0000 \\
\hline
\end{tabular}

TABLE 6: Predictive performance evaluation profiles of LHV for the testing phase by LHV-SIX.

\begin{tabular}{lcccccc}
\hline $\begin{array}{l}\text { Evaluation } \\
\text { indicators }\end{array}$ & $\begin{array}{c}\text { Minimum of } \\
\text { indicators }\end{array}$ & $\begin{array}{c}\text { Maximum of } \\
\text { indicators }\end{array}$ & $\begin{array}{c}\text { Mean of } \\
\text { indicators }\end{array}$ & $\begin{array}{c}\text { Median of } \\
\text { indicators }\end{array}$ & $\begin{array}{c}\text { Standard deviation of } \\
\text { indicators }\end{array}$ & Variance of indicators \\
\hline MAE & 46.5489 & 56.1094 & 49.5053 & 48.8671 & 1.9494 & 3.8002 \\
RMSE & 66.7362 & 80.9819 & 71.9365 & 71.4058 & 2.7337 & 7.4731 \\
R-square & 0.9783 & 0.9853 & 0.9829 & 0.9831 & 0.0013 & 0.0000 \\
EVS & 0.9797 & 0.9854 & 0.9837 & 0.9838 & 0.0010 & 0.0000 \\
\hline
\end{tabular}

value and the final value of valid loss for 100 trials, and those are illustrated in Figure 12. However, the above flaw almost disappears while aiming at LHV-SIX. This demonstrates the inadequacies and flaws of the ANN model sufficiently. The applicability and stability of the FEB-LCR-ACM need to be made further targeted performance improvements.

Nevertheless, one flaw cannot obscure the splendor of the jade. The FEB-LCR-ACM shows a good predictive performance based on the easily available hardware platform that is described in Section 5.3. On the one side, the time consumed is between 87.088 seconds and 95.563 in every experiment for LHV-IVE, and the average elapsed time is 89.098 ones. For 100 experiments, the standard deviation and variance of the experimental time are only 1.387 and 1.925, respectively. On the other side, the consuming time is between 106.640 seconds and 115.400 seconds in every experiment for LHV-SIX, and the average consuming time is 109.794 seconds. For 100 experiments, the standard deviation and variance of the experimental time are 1.533 and 2.349, respectively. All the computing time statistics show the good lightweight design paradigm and outstanding adaptive evolutionary architecture of FEBLCR-ACM.

In fact, the abovementioned statistics demonstrates the operational performance of the FEB-LCR-ACM, which is highly efficient, tranquil, and stable. It means that the FEBLCR-ACM for the specific CTHS can acquire the minute-

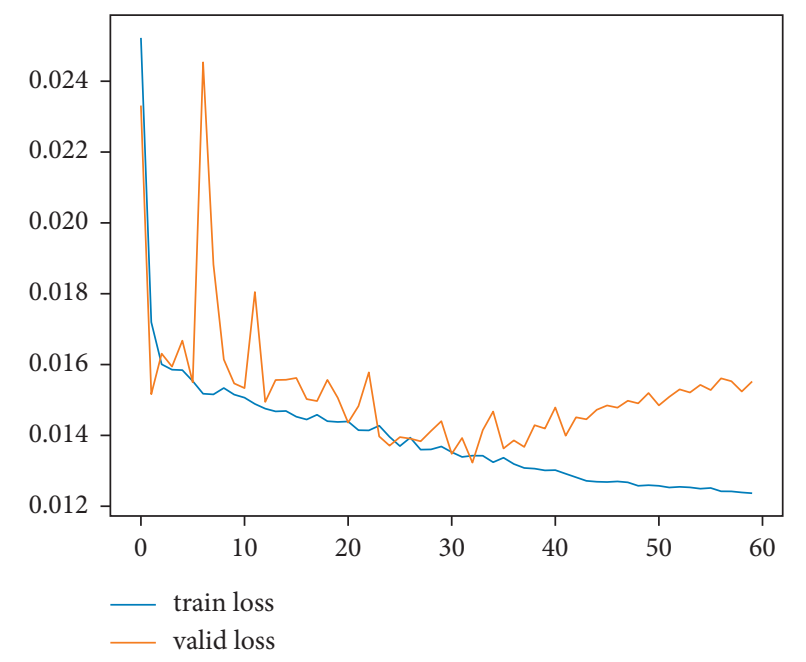

FIgURE 11: A deficient FEB-LCR-ACM validating loss curve by LHV-IVE.

level and quasi-real-time interaction between the DNN model and physical CTO-LGC. That is to be completely in conformity with the requirements of theory and practice for FEB-LCR-ACM. It has laid a good foundation for the practical application of FEB-LCR-ACM because of the acceptable computational complexity, computing expense, and response time. 


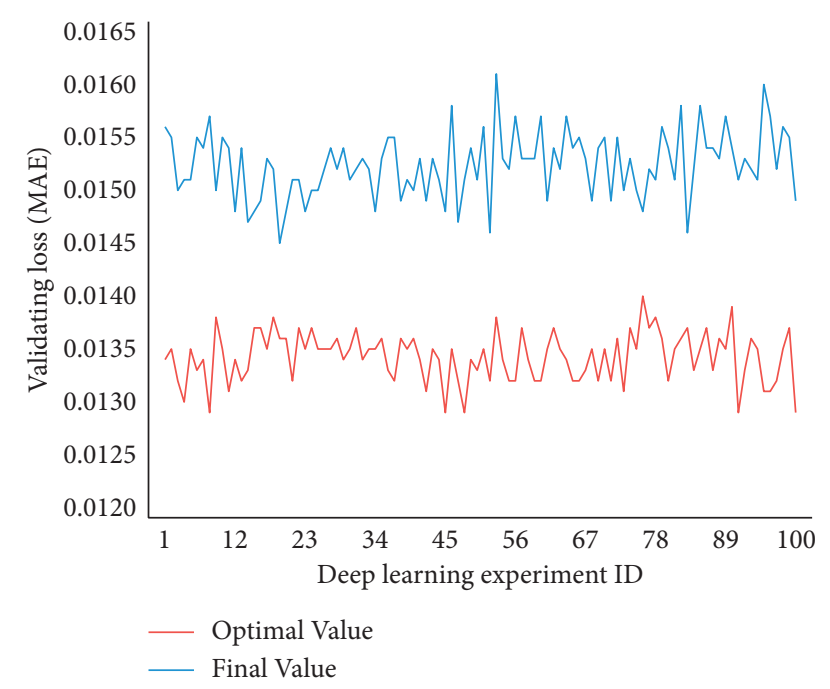

FIgURE 12: A comparison of optimal value and final value for validating loss by LHV-IVE.

\section{Conclusions}

This paper focuses on the automation and intelligence of CTO-LGC by proposing the FEB-LCR-ACM to support the PSAC decision at container terminals. The FEB-LCR-ACM is an automation mechanism, feature engineering, computing architecture, design paradigm, and learning philosophy essentially by the combination and integration of computational logistics and deep learning. The FEB-LCR-ACM is an important component of the brain center for CTHS that plays a key role in self-learning, auto tuning, and adaptive evolution. The FEB-LCR-ACM is supposed to drive, achieve, and fulfill the mechanization, automation, and intelligence of CTOLGC. The FEB-LCR-ACM is a specific practice of containerterminal-oriented neural-physical fusion computation substantially [60], which is the integration and synergy between computational logistics and deep learning through the cyberphysical systems as a bridge in essence. Moreover, it also provides a unified insight and an efficient solution to exploring and exploiting the discrete optimization management in complex and smart logistics and transportation systems under dynamic and uncertain environments.

\section{Data Availability}

The data used to support the findings of this study are included within the article.

\section{Conflicts of Interest}

The authors declare no conflicts of interest.

\section{Acknowledgments}

This research was funded by the Humanities and Social Science Programming Foundation of Ministry of Education in China, grant number (19YJA630031), the Scientific Research Foundation of Fujian University of Technology in China, grant number (GY-Z160125), and the Traffic Science and Technology Project of Shandong Province in China, grant number (2016B35).

\section{References}

[1] Ç. Iris and J. S. L. Lam, "A review of energy efficiency in ports: operational strategies, technologies and energy management systems," Renewable and Sustainable Energy Reviews, vol. 112, pp. 170-182, 2019.

[2] B. G. Zweers, S. Bhulai, and R. D. van der Mei, "Pre-processing a container yard under limited available time," Computers \& Operations Research, vol. 123, Article ID 105045, 2020.

[3] M. Neagoe, H.-H. Hvolby, M. Sadegh Taskhiri, and P. Turner, "Using discrete-event simulation to compare congestion management initiatives at a port terminal," Simulation Modelling Practice and Theory, vol. 112, Article ID 102362, 2021.

[4] Z. Lu, D. Zhuge, L. Murong, R. Yan, and S. Wang, "Operation management of green ports and shipping networks: overview and research opportunities," Frontiers of Engineering Management, vol. 6, no. 2, pp. 152-162, 2019.

[5] J. X. Luo, "Fully automatic container terminals of Shanghai Yangshan port phase IV," Frontiers of Engineering Management, vol. 6, no. 3, pp. 457-462, 2019.

[6] S. Si, J. Zhao, Z. Cai, and H. Dui, "Recent advances in system reliability optimization driven by importance measures," Frontiers of Engineering Management, vol. 7, no. 3, pp. 335358, 2020.

[7] S. Wang, Z. Lu, and N. Harilaos, "Three potential benefits of the EU and IMO's landmark efforts to monitor carbon dioxide emissions from shipping," Frontiers of Engineering Management, vol. 8, no. 2, pp. 310-311, 2021.

[8] H. L. Lee and Z.-J. Shen, "Supply chain and logistics innovations with the belt and road initiative," Journal of Management Science and Engineering, vol. 5, no. 2, pp. 77-86, 2020.

[9] Z. Lu, S. Wang, and K. Wang, "Terminal allocation problem in a transshipment hub considering bunker consumption," Naval Research Logistics, vol. 63, no. 7, pp. 529-548, 2016.

[10] Z. Lu, Y. Hu, S. Wang, G. Laporte, and Y. Wu, "Fleet deployment and demand fulfillment for container shipping liners," Transportation Research Part B: Methodological, vol. 120, pp. 15-32, 2019.

[11] Z. Lu, K. Wang, S. Wang, and X. Qu, "Tug scheduling for hinterland barge transport: a branch-and-price approach," European Journal of Operational Research, vol. 265, no. 1, pp. 119-132, 2018.

[12] G. L. Kumawat, D. Roy, R. De Koster, and I. Adan, "Stochastic modeling of parallel process flows in intra-logistics systems: applications in container terminals and compact storage systems," European Journal of Operational Research, vol. 290, no. 1, pp. 159-176, 2021.

[13] S. Y. Huang and Y. Li, "A bounded two-level dynamic programming algorithm for quay crane scheduling in container terminals," Computers \& Industrial Engineering, vol. 123, pp. 303-313, 2018.

[14] M. Stojaković and E. Twrdy, "A decision support tool for container terminal optimization within the berth subsystem," Transport, vol. 31, no. 1, pp. 29-40, 2016.

[15] Y. Peng, X. Li, W. Wang, K. Liu, and C. Li, “A simulationbased research on carbon emission mitigation strategies for green container terminals," Ocean Engineering, vol. 163, pp. 288-298, 2018. 
[16] I. Castilla-Rodríguez, C. Expósito-Izquierdo, B. MeliánBatista, R. M. Aguilar, and J. Marcos Moreno-Vega, "Simulation-optimization for the management of the transshipment operations at maritime container terminals," Expert Systems with Applications, vol. 139, Article ID 112852, 2020.

[17] J. M. Wing, "Computational thinking," Communications of the ACM, vol. 49, no. 3, pp. 33-35, 2006.

[18] P. J. Denning, "Beyond computational thinking," Communications of the ACM, vol. 52, no. 6, pp. 28-30, 2009.

[19] K. Kirkpatrick, "Parallel computational thinking," Communications of the ACM, vol. 60, no. 12, pp. 17-19, 2017.

[20] Y. Osman, "A new perspective on computational thinking," Communications of the ACM, vol. 61, no. 7, pp. 33-39, 2018.

[21] J. Schmidhuber, "Deep learning in neural networks: an overview," Neural Networks, vol. 61, pp. 85-117, 2015.

[22] V. Sharma and R. N. Mir, "A comprehensive and systematic look up into deep learning based object detection techniques: a review," Computer Science Review, vol. 38, Article ID 100301, 2020.

[23] T. van Klompenburg, A. Kassahun, and C. Catal, "Crop yield prediction using machine learning: a systematic literature review," Computers and Electronics in Agriculture, vol. 177, Article ID 105709, 2020.

[24] M. Bertolini, D. Mezzogori, M. Neroni, and F. Zammori, "Machine learning for industrial applications: a comprehensive literature review," Expert Systems with Applications, vol. 175, Article ID 114820, 2021.

[25] B. Zhao, X. Li, X. Lu, and Z. Wang, "A CNN-RNN architecture for multi-label weather recognition," Neurocomputing, vol. 322, pp. 47-57, 2018.

[26] M. Usama, B. Ahmad, E. Song, M. Shamim Hossain, M. Alrashoud, and G. Muhammad, "Attention-based sentiment analysis using convolutional and recurrent neural network," Future Generation Computer Systems, vol. 113, pp. 571-578, 2020.

[27] Y. Zhang, H. Lin, Z. Yang et al., "Neural network-based approaches for biomedical relation classification: a review," Journal of Biomedical Informatics, vol. 99, Article ID 103294, 2019.

[28] A. Thakkar and K. Chaudhari, "A comprehensive survey on deep neural networks for stock market: the need, challenges, and future directions," Expert Systems with Applications, vol. 177, Article ID 114800, 2021.

[29] R. Zhao, R. Yan, Z. Chen et al., "Deep learning and its applications to machine health monitoring," Mechanical Systems and Signal Processing, vol. 115, pp. 213-237, 2019.

[30] H. Bi, T. Mao, Z. Wang, and Z. Deng, "A deep learning-based framework for intersectional traffic simulation and editing," IEEE Transactions on Visualization and Computer Graphics, vol. 26, no. 7, pp. 2335-2348, 2020.

[31] L. Monostori, V. Paul, D. Alexandre, H. Panetto, M. Brdys, and B. C. Csáji, "Cooperative control in production and logistics," Annual Reviews in Control, vol. 39, pp. 12-29, 2015.

[32] S.-H. Chung, H.-L. Ma, M. Hansen, and T.-M. Choi, "Data science and analytics in aviation," Transportation Research Part E: Logistics and Transportation Review, vol. 134, Article ID 101837, 2020.

[33] B. Yu, Z. Guo, A. Sobhan, H. Wang, and G. Chen, "Flight delay prediction for commercial air transport: a deep learning approach," Transportation Research Part E: Logistics and Transportation Review, vol. 125, pp. 203-221, 2019.

[34] S. Ren, C. Tsan-Ming, K.-M. Lee, and L. Lei, "Intelligent service capacity allocation for cross-border-E-commerce related third-party-forwarding logistics operations: a deep learning approach," Transportation Research Part E: Logistics and Transportation Review, vol. 134, Article ID 101834, 2020.

[35] P. Sushil, N. Konstantinos, S. S. Prakash, J. K. Madaan, and L. Konstantia, "Deep learning with long short-term memory networks and random forests for demand forecasting in multi-channel retail," International Journal of Production Research, vol. 58, no. 16, pp. 4964-4979, 2020.

[36] H. Shen and J. Lin, "Investigation of crowdshipping delivery trip production with real-world data," Transportation Research Part E: Logistics and Transportation Review, vol. 143, Article ID 102106, 2020.

[37] Y.-T. Chen, E. W. Sun, M.-F. Chang, and Y.-B. Lin, "Pragmatic real-time logistics management with traffic IoT infrastructure: big data predictive analytics of freight travel time for Logistics 4.0," International Journal of Production Economics, vol. 238, Article ID 108157, 2021.

[38] H. Guo, G. Cheng, B. Xu, Y. Xia, and F. Sun, "MLP neural network-based regional logistics demand prediction," Neural Computing \& Applications, vol. 33, no. 9, pp. 3939-3952, 2021.

[39] L. Huang, G. Xie, W. Zhao, Y. Gu, and Y. Huang, "Regional logistics demand forecasting: a BP neural network approach," Complex \& Intelligent Systems, 2021.

[40] P. Boutselis and K. McNaught, "Using Bayesian networks to forecast spares demand from equipment failures in a changing service logistics context," International Journal of Production Economics, vol. 209, pp. 325-333, 2019.

[41] T. Funke and T. Becker, "Complex networks of material flow in manufacturing and logistics: modeling, analysis, and prediction using stochastic block models," Journal of Manufacturing Systems, vol. 56, pp. 296-311, 2020.

[42] C. Pani, P. Fadda, G. Fancello, L. Frigau, and F. Mola, "A data mining approach to forecast late arrivals in a transhipment container terminal," Transport, vol. 29, no. 2, pp. 175-184, 2014.

[43] Y. Gao, C. Chun-Hsien, D. Chang, and F. Ting, "Deep learning with long short-term memory recurrent neural network for daily container volumes of storage yard predictions in port," in Proceedings of the 17th International Conference on Cyberworlds, pp. 427-430, IEEE, Singapore, October 2018.

[44] S. Sonali, I. P. Vigneswara, P. Sushil, and S. S. Prakash, "Forecasting container throughput with long short-term memory networks," Industrial Management \& Data Systems, vol. 120 , no. 3, pp. 425-441, 2020.

[45] B. Liu, "New technology application in logistics industry based on machine learning and embedded network," Microprocessors and Microsystems, vol. 80, Article ID 103596, 2021.

[46] Y. Liu, L. Yan, S. Li et al., "Enhancing input parameter estimation by machine learning for the simulation of large-scale logistics networks," in Proceedings of the 2020 Winter Simulation Conference, pp. 608-619, Orlando, FL, USA, December 2020.

[47] E. Göçmen and R. Erol, "Transportation problems for intermodal networks: mathematical models, exact and heuristic algorithms, and machine learning," Expert Systems with Applications, vol. 135, pp. 374-387, 2019.

[48] X. Dai, M. Chen, and Y. Zhou, "Optimal logistics transportation and route planning based on FPGA processor realtime system and machine learning," Microprocessors and Microsystems, vol. 80, Article ID 103621, 2021.

[49] X. Xu, Y. Shen, W. Chen, Y. Gong, and H. Wang, "Datadriven decision and analytics of collection and delivery point 
location problems for online retailers," Omega, vol. 100, Article ID 102280, 2021.

[50] M. Andreas, F. Johannes, and T. Jansen, "Data-driven deep learning for proactive terminal process management," in Proceedings of the Industry Forum at Business Process Management 2019, pp. 190-201, Vienna, Austria, September 2019.

[51] C. Zhang, H. Guan, Y. Yuan, W. Chen, and T. Wu, "Machine learning-driven algorithms for the container relocation problem," Transportation Research Part B: Methodological, vol. 139, pp. 102-131, 2020.

[52] H. André, S. Tanaka, and T. Kevin, “Deep learning assisted heuristic tree search for the container pre-marshalling problem," Computers and Operations Research, vol. 113, Article ID 104781, 2020.

[53] B. Li, "Collection and distribution virtual machines architecture of container terminal based on computational logistics and its simulation analysis," Computer Integrated Manufacturing Systems, vol. 24, no. 1, pp. 245-263, 2018.

[54] A. Gharehgozli, N. Zaerpour, and R. de Koster, "Container terminal layout design: transition and future," Maritime Economics \& Logistics, vol. 22, pp. 610-639, 2020.

[55] P. Wang, P. Joan, Mileski, and Q. Zeng, "Alignments between strategic content and process structure: the case of container terminal service process automation," Maritime Economics \& Logistics, vol. 21, pp. 543-558, 2019.

[56] T. Jonker, M. B. Duinkerken, N. Yorke-Smith, A. de Waal, and R. R. Negenborn, "Coordinated optimization of equipment operations in a container terminal," Flexible Services and Manufacturing Journal, vol. 33, pp. 281-311, 2021.

[57] B. Li, B. Sun, W. Yao, Y. He, and G. Song, "Container terminal oriented logistics generalized computational complexity," IEEE Access, vol. 7, pp. 94737-94756, 2019.

[58] B. Li, "Container terminal logistics scheduling and decisionmaking within the conceptual framework of computational thinking," in Proceedings of the IEEE 54th Annual Conference on Decision and Control (CDC 2015), pp. 330-337, IEEE, Osaka, Japan, December 2015.

[59] B. Li and G. Song, "Computational logistics for container terminal hubs based on computational lens and computing principles," IEEE Access, vol. 8, pp. 194820-194835, 2020.

[60] B. Li and Y. He, "Computational logistics for container terminal handling systems with deep learning," Computational Intelligence and Neuroscience, vol. 2021, Article ID 5529914, 18 pages, 2021.

[61] M. Mohammadi, A. Al-Fuqaha, S. Sorour, and M. Guizani, "Deep learning for IoT big data and streaming analytics: a survey," IEEE Communications Surveys \& Tutorials, vol. 20, no. 4, pp. 2923-2960, 2018.

[62] B. Li and Y. He, "Container terminal liner berthing time prediction with computational logistics and deep learning," in Proceedings of the 2020 IEEE International Conference on Systems, Man, and Cybernetics (SMC 2020), pp. 2417-2424, IEEE, Toronto, Canada, October 2020.

[63] B. Li and Y. He, "An attention mechanism oriented hybrid CNN-RNN deep learning architecture of container terminal liner handling conditions prediction," Computational Intelligence and Neuroscience, vol. 2021, Article ID 3846078, 17 pages, 2021.

[64] B. Li, "Hierarchical, parallel, heterogeneous and reconfigurable computation model of container terminal handling system," Journal of Traffic and Transportation Engineering, vol. 19, no. 2, pp. 136-155, 2019.

[65] M. Christ, N. Braun, J. Neuffer, and W. Andreas, "Time series FeatuRe extraction on basis of scalable hypothesis tests (tsfresh-a Python package)," Neurocomputing, vol. 307, pp. 72-77, 2018.

[66] B. Yuan, Y. J. Tan, K. Mudunuru Maruti et al., "Using machine learning to discern eruption in noisy environments: a case study using $\mathrm{CO}_{2}$-driven cold-water geyser in Chimayó, New Mexico," Seismological Research Letters, vol. 90, no. 2A, pp. 591-603, 2019.

[67] F. Wang, D. Zhang, Z. Li, and X. Zhao, "Method for sEMGbased motion recognition for patients at different brunnstrom stages," Robot, vol. 42, no. 6, pp. 661-685, 2020.

[68] G. Liu, L. Li, L. Zhang, Q. Li, and S. S. Law, "Sensor faults classification for SHM systems using deep learning-based method with Tsfresh features," Smart Materials and Structures, vol. 29, no. 7, Article ID 075005, 2020.

[69] H. Ehya, T. Skreien, and A. Nysveen, "Intelligent data-driven diagnosis of incipient inter-turn short circuit fault in field winding of salient pole synchronous generators," IEEE Transactions on Industrial Informatics, 2021.

[70] Z. Yang, A. I. Ahmed, M. E. Elmubarak, S. Ali, and M. Zhang, "An anomaly detection algorithm selection service for IoT stream data based on tsfresh tool and genetic algorithm," Security and Communication Networks, vol. 2021, Article ID 6677027, 10 pages, 2021.

[71] X. Luo, J. Sun, L. Wang et al., "Short-term wind speed forecasting via stacked extreme learning machine with generalized correntropy," IEEE Transactions on Industrial Informatics, vol. 14, no. 11, pp. 4963-4971, 2018.

[72] T. Hu, Q. Guo, Z. Li, X. Shen, and H. Sun, "Distribution-free probability density forecast through deep neural networks," IEEE Transactions on Neural Networks and Learning Systems, vol. 31, no. 2, pp. 612-625, 2020.

[73] G. Song, B. Li, and Y. He, "Container terminal logistics generalized computing architecture and green initiative computational pattern performance evaluation," Information, vol. 10 , no. 12 , p. $383,2019$.

[74] L. J. Cao and F. E. H. Tay, "Support vector machine with adaptive parameters in financial time series forecasting," IEEE Transactions on Neural Networks, vol. 14, no. 6, pp.1506-1518, 2003.

[75] I. D. da Silva, M. C. Moura, I. D. Lins, E. López Droguett, and E. Braga, "Non-stationary demand forecasting based on empirical mode decomposition and support vector machines," IEEE Latin America Transactions, vol. 15, no. 9, pp. 1785-1792, 2017. 